\title{
Effects of miRNAs on functions of breast cancer stem cells and treatment of breast cancer
}

This article was published in the following Dove Press journal:

OncoTargets and Therapy

\author{
Ying Zhang ${ }^{1, *}$ \\ Bin $X u^{2, *}$ \\ Xi-ping Zhang ${ }^{3}$ \\ 'Department of Breast Surgery, \\ Fudan University Shanghai Cancer \\ Center, Shanghai, China; ${ }^{2}$ Department \\ of Surgery, Zhejiang Rehabilitation \\ Medical Center, Hangzhou, China; \\ ${ }^{3}$ Department of Breast Surgery, \\ Zhejiang Cancer Hospital, Hangzhou, \\ Zhejiang Province, China \\ *These authors contributed equally \\ to this work
}

\begin{abstract}
Breast cancer is one of the most common malignancies for women, which accounts for $30 \%$ of all female malignancies. The formation of breast cancer stem cells (BCSCs) is attributed to the acquisition of stemness of tumor cells. With self-renewal potential, these stem cells are insensitive to either radiotherapy or chemotherapy but are significant in regulating tumor behaviors and drug resistance. MicroRNA (miRNA) is a kind of noncoding small RNA for negatively regulating gene expressions. Research findings suggest that many miRNAs specifically regulate the expression of target genes and signal pathways of BCSCs. They play an important role in self-renewal, growth, and metastasis of breast cancer cells as potential targets for treating breast cancer. These signal pathways include phosphatase and tensin homolog deleted on chromosome 10-phosphatidylinositol 3-kinase/Akt, Wnt/ $\beta$-catenin, Notch, and so on. This paper reviews the progress of research about miRNAs in self-renewal, metastasis, epithelial-mesenchymal transition and metastasis, mediation of resistance to chemotherapies, and treatment of breast cancer.
\end{abstract}

Keywords: breast cancer, stem cells, microRNA, EMT

\section{Introduction}

Tumorigenic stem cells only make up a small proportion of numerous stem cells in breast cancer tissues. ${ }^{1}$ These stem cells have abilities to develop into other tumorigenic and nontumorigenic cells, which are known as breast cancer stem cells (BCSCs). In BCSCs, CD44, as a surface marker, is highly expressed, whereas CD24 is lowly or not expressed. ${ }^{2}$ Both CD24 and CD44 get involved in biologic behaviors of tumor cells, including adhesion, migration, and metastasis. ${ }^{3,4}$ High expression of aldehyde dehydrogenase 1 is another feature of BCSCs. ${ }^{5}$ Instead of a single population transformed from ordinary stem cells, BCSCs are attributable to the stemness of tumor cells. ${ }^{6}$ Previous studies have discovered that more BCSCs are seen in poorly differentiated and highly malignant breast cancer tissues than highly differentiated, low-grade malignancies. ${ }^{7}$ BCSCs are insensitive to radiotherapies and chemotherapies, no matter in vivo or vitro. ${ }^{8}$ After adjuvant therapies, BCSCs increase considerably, ${ }^{9,10}$ thus possibly leading to recurrence and metastasis of breast cancer. Hence, research on BCSCs is of great significance for treating breast cancer.

As noncoding RNA molecules with about 22 nucleotides, microRNAs (miRNAs) complementarily bind with $3^{\prime}$ noncoding region of target RNAs, to degrade target mRNAs or inhibit their translations and negatively regulate gene expressions. ${ }^{11}$ MiRNAs main function is to posttranscriptionally downregulate gene expression by binding directly to its mRNA target or by cleaving target mRNA by binding to its 3 '-untranslated region (UTR) region. MiRNAs are closely connected with formation 
and development of tumors and cancer stem cells (CSCs). Because of amplification, each miRNA can increase the control over its target gene. If the target gene is an oncogene, the cancer does not develop (oncosuppressor-miRs); if the target gene is a tumor suppressor, the cancer develops (oncomiRs). ${ }^{12}$ MiRNAs play a pivotal role in stem cell biology, differentiation, and oncogenesis, and are of high interest as potential breast cancer therapeutics. However, their expression and function during normal mammary differentiation and in breast cancer remain to be elucidated. ${ }^{13}$

These years, regulating BCSCs by miRNAs has become one of the hot research topics on breast cancer. For instance, miR-221/222, miR-140, miR-21, and miR-22 are carcinogenic, whereas miR-200 family, miR-128, miR-99a, miR-29b, let-7 miRNA, miR-600, miR-34, and miR-30 are effective for tumor suppression. Secreted in the form of exosomes, miRNAs play an important role in transmitting signals between cells. ${ }^{14}$ This paper reviews research progression of miRNAs in self-renewal, metastasis, epithelial-mesenchymal transition (EMT) and metastasis, mediation of resistance to chemotherapies, and treatment of breast cancer.

\section{MiRNAs, self-renewal, and apoptosis of BCSCs}

\section{B lymphoma Mo-MLV insertion region I homolog (BMII), a self-renewal factor}

BMI1 is a member of the polycomb repression complex 1 family and regulates the self-renewal of normal and malignant stem cells by preventing senescence and apoptosis. BMI1 is an independent prognostic factor of triple-negative breast cancer (TNBC) and is essential for the self-renewal of BCSCs. BMI1 was shown to be overexpressed in several human breast cancer cell lines. ${ }^{15}$ By regulating the expressions of BMI1 and Suz12 (polycomb repressive complex 2 subunit) that are involved in self-renewal of BCSCs, miR-200c could suppress the colony formation of BCSCs in vitro and the tumor formation induced by stem cells in vivo. ${ }^{16}$ High expression of miR-200c was associated with significantly better overall survival. ${ }^{17}$ miR-200c, as a tumor suppressor in breast cancer both in vitro and in vivo, could be a potential therapeutic target in breast cancer. ${ }^{18}$ When the expression of miR-200 is downregulated, mammary epithelial cells will transform to stem-cell-like phenotypes. ${ }^{19}$ Through ectopic expression of BCSCs, miR-128 may lower the expression of BMI1 and ABCC5, inhibit breast cancer progression, and induce apoptosis in vivo and vitro. ${ }^{20}$

\section{PTEN-PI3K/Akt pathway}

Phosphatase and tensin homolog deleted on chromosome 10 (PTEN) protein, which activates lipid phosphatase and protein phosphatase, suppresses cancer through phosphorylating and targeting downstream molecules of phosphatidylinositol 3-kinase (PI3K) to block PI3K/Akt pathway. Once it is ectopically expressed through the PTEN-PI3K/Akt pathway, miR-221/222 may increase BCSCs, enhance tumorforming abilities of breast cancer cells, suppress expression of recoverable PTEN of endogenous miR221/222, inhibit Akt phosphorylation, and reverse the acquisition of cell stemness. ${ }^{21}$ MiR-99a targets the downstream factor mechanistic target of rapamycin kinase and the downstream gene HIF-1 $\alpha$ (hypoxia-inducible factor 1 alpha subunit) of PI3K/ $\mathrm{Akt}$, in order to reverse phenotypes of malignant BCSCs. ${ }^{22}$ When R273H of P53 mutates, miR-30a would not inhibit the expression of IGF-1R (insulin-like growth factor 1 receptor) anymore, but activate the AKT (serine/threonine kinase) signaling pathway. Further suppression of miR-30a would slow down or reverse tumor progression of p53R $273 \mathrm{H} .{ }^{23}$ Under nonadherent conditions, miR-30a may negatively regulate gene expressions of protein AVEN (apoptosis and caspase activation inhibitor) to inhibit the growth of BCSCs. ${ }^{24}$

\section{Wnt $/ \beta$-catenin signal pathway}

$\mathrm{Wnt} / \beta$-catenin pathway is a signal pathway for regulating differentiation of human stem cells, ${ }^{25}$ playing a crucial regulatory role in multiple development stages of mammary glands and serving the important functions for stabilizing the quantity of BCSCs. ${ }^{26,27}$ During the progression of breast cancer, estrogenic elements and abnormal methylation of $\mathrm{CpG}$ islands in the promoter region of miR-140 disinhibit pathways regulating BCSCs such as Wnt, SOX2 (sex-determining region Y-box 2), and SOX9 (sex-determining region Y-box 9). Thus, carcinoma in situ may progress to infiltrating cancer. ${ }^{28}$ For TNBC, the expression level of miR-29b is negatively correlated to the potential of BCSCs. Apart from inhibiting the expression of SPIN1 (spindlin 1), miR-29b may also inhibit growth, self-renewal, and invasion of TNBC cells by suppressing Wnt/ $\beta$-catenin and AKT signal pathways. ${ }^{29} \mathrm{Yu}$ et al have discovered that the expression of let-7 is downregulated in BCSCs. ${ }^{30}$ In vitro, let-7 directly inhibits H-RAS (HRas protooncogene) and HMGA2 (high-mobility group AT-hook 2), which are related to self-renewal and pluripotent potential of stem cells. In immunodeficient mouse models, growth of tumor cells and oncogenesis will be inhibited if the expression of let-7 (microRNA let-7) is induced. When it targets oncogenes such as RAS (Ras oncogene) and MYC (MYC protooncogene), let-7 has feedback effects upon LIN28, which is a downstream gene of $\mathrm{Wnt} / \beta$-catenin pathway, thus inhibiting itself from forming loops. ${ }^{31,32}$ It can maintain stemness and facilitate metastasis of tumors for HER2-positive and TNBC. ${ }^{33}$ 
In addition, miR-600 targets SCD1 enzyme that gets involved in the modification of WNT proteins, thereby inhibiting the activity of $\mathrm{Wnt} / \beta$-catenin pathways and promoting differentiation of BCSCs. ${ }^{34}$ In Bodal et al's study, miR-146 variant allele was found to be marginally associated with increased breast cancer risk. ${ }^{35}$ Other research also reported similar association of pre-miR-146 single-nucleotide polymorphism with cancer risk. MiR-146b can be the promising markers for differential diagnostics of various phenotypes of breast cancer. ${ }^{36}$ MiR-146a and miR-146-5p silence BRCA1 gene through binding to its 3 '-UTR, accompanied by a reduced homologous recombination rate and an increased proliferation, thus triggering the formation of triple-negative and basal-like sporadic BC cases. ${ }^{37}$ Downregulation of miR-146a and miR-146b expression in breast tissues was related to the development and deterioration of breast cancer. MiR-146a and miR-146b might act as potential biomarkers for young women with breast cancer. ${ }^{38} \mathrm{MiR}-146 \mathrm{a}$, an miRNA upregulated in human BCSCs, also activates the Notch signal pathway by targeting Numb, a suppressor of the Notch signal pathway. Some findings suggest that the downregulation of miR-200 members and the upregulation of miR-146 are involved in the activation of the Notch signal pathway in the BCSCs and normal mammary stem/progenitor cells. ${ }^{39}$

\section{Notch signal pathway}

Notch signal pathway is one of the important signal pathways for regulating self-renewal and apoptosis of BCSCs. It is associated with the regulation of cell fate at several distinct developmental stages of the mammary gland and has been implicated in cancer initiation and progression. ${ }^{40}$ So far, four Notch receptors (Notch1-4) have been discovered in vertebrates. Once they are bound to Delta-Serrate LAG2, Notch receptors are split to activate the signal pathway. The intracellular domain of Notch enters the cell nucleus after it falls off cell membrane and interacts with DNA-binding proteins of the CSL family, to induce transcription of target genes. By specific inhibition of the Notch pathway, it is possible to significantly reduce the number of BCSCs and brain metastasis of breast cancer. ${ }^{41}$ In the MCF-7 cell line, miR-34a downregulates the Notch1 receptor, inhibits stemness of breast cancer, and makes breast cancer cells more sensitive to paclitaxel by inhibiting the Notch pathway. ${ }^{42}$ miR-200 family miRNAs suppress the Notch signaling by targeting Notch pathway components, such as JAG1 and the mastermind-like Notch co-activators, Maml2 and Maml3. 39,43 Moreover, overexpression of Notch signaling can also be suppressed by the induction of miR-9 and miR-34c expression, thus reducing metastatic behaviors in TNBC. ${ }^{44}$

\section{The characteristics of BCSCs and normal mammary stem cells}

Understanding the similarity and difference of stem cell properties between human BCSCs and normal mammary stem cells will clarify the roles of BCSCs in human breast cancer development and progression. ${ }^{39}$ Mammary gland stem cells have self-renewal ability, which is the basis for producing tubular and lobular structure of the mammary glands and can differentiate into all the series of mammary epithelial cells. Breast stem cells guarantee the maintenance of regeneration, lactation, and degenerative cycle of mammary gland. Mammary gland stem cells are thought to have multiple functions in regulating mammary gland development, tissue maintenance, major growth, and structural remodeling. In addition, accumulative evidence suggests that breast cancers are initiated and maintained by a subpopulation of tumor cells with stem cell features (called CSCs). ${ }^{45}$

BCSCs are the first CSCs prospectively identified from human solid tumors. BCSCs and normal mammary stem cells share a part of the genetic and epigenetic properties that are associated with the regulation of tissue stem cells. ${ }^{39}$ To maintain tissue homeostasis, normal stem cells must be able to undergo a large number of mitoses, and in many tissues, they must be able to migrate to different regions of the organ. Both properties are reminiscent of two hallmark properties of cancer cells, immortality and invasion. The functional similarities of cancer cells with normal tissue stem cells suggest that activation of normal stem cell self-renewal and/or differentiation pathways accounts for many of the properties associated with malignancies. ${ }^{16}$ The characteristics of BCSCs are as follows 1) they have unlimited self-renewal ability, which can generate heterogeneous tumor cells; 2) they have the ability of multipotential differentiation and high tumorigenicity; 3) they have the drug resistance to conventional chemotherapeutics; and 4) they have the ability of strong migration and invasion. ${ }^{46}$

\section{Others}

MiR-34c, with low expressions in BCSCs of MCF-7 and SK-3rd cell lines, induces self-renewal of cells through hypermethylation in the promoter region of BCSCs. ${ }^{43}$ The downregulation of miR-30, particularly miR-30e, often contributes to stemness acquisition by breast cancer tissues. The high expression of miR-30e could inhibit self-renewal abilities of BSCSs by suppressing the expression of Ubc9 (hypothetical protein) and apoptosis caused by ITGB3 (integrin subunit beta 3 ) silencing. Besides, experiments with mice have demonstrated that low expression of miR-30 is fairly important for maintaining self-renewal and antiapoptotic properties of BCSCs. ${ }^{47}$ 
To sum up, signal pathways regulating self-renewal and differentiation of stem cells such as Wnt/ $\beta$-catenin, Notch, PTEN-PI3K/Akt, and BMI1 pathways often exhibit abnormal expressions in breast cancer, leading to the acquisition of stem cell phenotypes while developing potentials of selfrenewal, differentiation, and antiapoptosis. ${ }^{48}$

\section{Epigenetic regulation over the effects of BCSCs for promoting EMT by miRNAs}

CSCs have features necessary for inducing tumor metastasis (for instance, they are tumor-derived, invasive, and metastatic), ${ }^{49}$ playing an important role in neoplasm recurrence and metastasis. ${ }^{50}$ EMT is often activated in the process of breast cancer invasion and metastasis. In this process, mammary epithelial cells lose the epithelial cell marker E-cadherin, intercellular junctions, and cell polarity, while cell mobility increases, all of which lead to tumor progression. ${ }^{51}$ Furthermore, EMT induction may make breast cancer cells acquire stem-cell-like properties, ${ }^{52}$ thus further impacting self-renewal and differentiation of breast cancer cells.

BCSCs stimulate EMT by releasing transforming growth factor beta (TGF- $\beta) .{ }^{52}$ Meanwhile, miR-200 family regulates self-renewal and EMT of BCSCs. ${ }^{53}$ In terms of breast cancer, this family often shows high expressions in epithelial cells, but low expressions in mesenchymal cells, positively correlated to the expression of E-cadherin. ${ }^{54}$ It consists of five members, including two conserved genomic clusters, miR-200a, miR-200b, miR-149, miR-200c, and miR-141. Of these members, miR-200b-200a and miR-200c-141 clusters exhibit low expressions in BCSCs. ${ }^{55}$ The high expression of SIRT1 inhibits miR-200a, which may cause the mesenchymal transition of normal mammary epithelial cells. ${ }^{56}$ While attacking BMI1, miR-200c inhibits the growth of normal mammary cells and reverses the tumorigenicity of BCSCs. ${ }^{16}$ In addition, it may regulate the expression of E-cadherin and EMT by inhibiting miR-200c. ${ }^{55}$ ZEB1 (zinc finger E-box binding homeobox 1) and ZEB2 (zinc finger E-box binding homeobox 2) have 17 conservative binding sites for miR-200 family altogether. Accordingly, miR-200 owns multiple sites for binding with ZEB1 and ZEB2, ${ }^{57}$ which thus play an important role in regulating EMT. ZEB1/miR-200 axis can interact with other pathways to regulate EMT and biology of BCSCs. ${ }^{58}$

EMT is a prerequisite for tumor metastasis. In BCSCs, miR-200 also acts on SUZ12. Composed of SUZ12 (SUZ12 polycomb repressive complex 2 subunit) and other proteins, PCR2 controls the expression of E-cadherin. miR-200/ SUZ12/E-cadherin axis is critical for maintaining BCSCs and regulating metastasis of breast cancer. Both the overexpression of miR-200b and the low expression of SUZ12 result in lower expression of E-caherin to inhibit oncogenesis, growth, and invasion while playing certain roles in preventing tumor recurrence in transplanted tumor models treated with chemotherapies. ${ }^{59}$ Stem-cell-like breast cancer cells will be transformed into non-stem-cell-like ones if miR-200 is retransferred to them, and their EMT will be reversed. ${ }^{19}$ Additionally, miR-200c may directly act on class III $\beta$-tubulin (TUBB3) to recover antimicrotubule effects of paclitaxel and reverse drug resistance. Han and some other researchers have discovered that the formation of stem-cell-like cells with EMT potential is associated with HIF- $1 \alpha$ and regulated by miR-21. ${ }^{60}$ MiR-21 inhibits the expression of PTEN and activates AKT/ERK1/2 pathways to promote EMT, ${ }^{61}$ mediating invasion and metastasis of breast cancer. ${ }^{62}$ In MCF-7 cell lines, EMT and level of HIF- $1 \alpha$ may be reversed and lowered by suppressing the expression of miR-21 in BCSCs. ${ }^{60}$ Concerning estrogen-receptor-positive breast cancer, miR-22 promotes EMT and metastasis through hypermethylation of miR-200 promoter and downregulation of ZEB1/2-induced expression of BCSCs. The high expressions of miR-22 in non-TNBC are significantly correlated to high-grade cancer cells and relatively poor prognosis of tumor cells. ${ }^{63}$

Compared with other molecular subtypes, TNBC has more BCSCs. The expression of miR-205 is downregulated in breast cancer cells, which is associated with the recurrence of TNBC. ${ }^{64}$ It also targets ZEB1 and ZEB2, producing synergistic effects with the miR-200 family. ${ }^{55}$ When Notch pathway is activated, hairy and enhancer of split 1 (HES1), an inhibitory factor, acts upon the miR-205 promoter and inhibits the expression of miR-205 to upregulate ZEB1/2 and then targets Notch2, thus affecting differentiation, EMT, and oncogenesis of breast cancer cells. ${ }^{65}$

Apart from regulating self-renewal of BCSCs as mentioned above, miR-34c's downregulation also promotes EMT and inhibits the expression of Notch4, thus suppressing tumor metastasis. In addition, E12 and E47, as transcription factors, are effective for upregulating the expression of miR-495 to inhibit the expression of E-cadherin while promoting stemness acquisition, invasion, and metastasis of breast cancer cells. ${ }^{66}$ By downregulating TGF- $\beta$ signal pathway and several genes regulating stem cells (including JAK1 [Janus kinase 1], AKT3 [serine/threonine kinase 3], STAT3 [signal transducer and activator of transcription 3], SOX4 [sex-determining region Y-box 4], EZH1 [enhancer of zeste 1 polycomb repressive complex 2 subunit], and HMGA2 [high mobility group AT-hook 2]), miR-93 reduces the number of BCSCs 
and reverses EMT. At the same time, its anticancer effects impact normal mammary stem cells as well. ${ }^{67}$

To sum up, miRNAs' signal pathways are somewhat associated with the stemness of stem cells and the acquisition of drug resistance, which is in accordance with the view that "EMT mediated by TGF- $\beta$ signal pathway may promote the expression of stem cell surface markers in normal breast cancer cells, which will thus acquire stemness". Both miR-200 family and miR-34 affect several different "carcinogenic" tendencies of BCSCs, which suggests that these miRNAs, with multiple roles against breast cancer, are possibly good tools for treatment. Furthermore, breast cancer with different molecular subtypes differs in the number of BCSCs and the corresponding miRNA expression profiles, which partially explains the reason why TNBC is more malignant.

\section{MiRNA-mediated resistance to chemotherapies}

CSCs could acquire the stemness from other mature tissues and cells after induction. ${ }^{68}$ With a low renewal rate, these stem cells may avoid the cytotoxic effects of chemotherapy targeting rapidly dividing cells, and thus mediate cancer recurrence. ${ }^{69}$ Existing therapies are not quite ideal for killing $\mathrm{CSCs},{ }^{10}$ so it is of great significance for curing breast cancer by killing BCSCs with miRNA. At present, mesenchymal stem cells (MSCs) that contain specific miRNAs are used for specifically targeting and attacking BCSCs for curing cancer, which has been one of the new research interests for treating breast cancer over the past 5 years.

Research suggests that drugs targeting drug-resistant miRNAs are potent for making breast cancer cells regain their sensitivity to chemotherapies. ${ }^{70}$ Directly inhibiting the expressions of BMI1 and ABCC5, miR-148 and miR-128 strengthen the effects of doxorubicin for promoting apoptosis and causing damages to DNA. ${ }^{20,71}$ For breast cancer, the low expression of miR-128 is associated with chemotherapy resistance and relatively poor prognosis of patients. By downregulating the expression of miR-128 in BCSCs, cancer cells will become more resistant to chemotherapies. ${ }^{71}$ Overexpression of miR-16 or inhibition of its target WIP1 may weaken self-renewal abilities of BCSCs in mice, thus making MCF7 sensitive to doxorubicin. ${ }^{72}$

As an integral part of tumor microenvironment, MSCs can be easily obtained and directly used without MHC pairing. Mature miRNAs or precursor RNAs may be transferred through exosomes. ${ }^{73}$ MSCs target tumor cells by releasing exosomes that contain miRNAs with such effects (e.g., miR-16). ${ }^{74}$ Hence, MSC is regarded as an ideal tool for transferring noncoding RNAs like miRNAs. Research has proven that co-culture of MSCs with exosomes of miR-23b and breast cancer cells may mediate tumor dormancy by suppressing MARCKS (myristoylated alanine rich protein kinase C substrate) genes that regulate cell cycle and motility. ${ }^{75}$ MiR-127, miR-197, miR-222, miR-223, ${ }^{76,77} \mathrm{miR}-21$, and miR-34a $\mathrm{a}^{78,79}$ have been also proven to play some roles in tumor cell dormancy, whereas MSC is communicating with breast cancer cells. This treatment strategy is still being studied. BCSCs are mostly distributed in hypoxic tumor regions. If tumor volume is too large, the efficiency of MSCs in transferring anticancer miRNAs would be impacted. So far, this problem is still unsolved. Another important question is whether normal cells are impacted while MSCs are excreting exosomes with miRNAs. These questions need to be further explored in future basic research.

\section{Future}

As more and more in-depth researches are performed, the biologic functions of miRNAs have been discovered more and more extensively, which would bring new hope for treating breast cancer. It has been discovered that miRNAs may create more chances for diagnosing and treating breast cancer in BCSCs. The utilization of miRNAs for treatment would weaken self-renewal abilities and antiapoptotic properties of BCSCs, and reverse drug resistance. By eradicating stem cells, progression, recurrence, and metastasis of breast cancer would be prevented. Some practices have demonstrated that the content of class-III $\beta$-tubulin will decline, and cytotoxicity of paclitaxel will significantly increase if solid lipid nanoparticles with miR-200c are integrated into BCSCs. ${ }^{80}$ Other molecular markers of BCSCs such as miR let-7, miR-16, miR-128, and miR-148 are also potential antitumor targets. ${ }^{81}$ It is a new therapeutic strategy for transferring miRNAs through MSCs and having them act on target BCSCs. The clinical safety and therapeutic effects of this strategy remain to be further explored.

Previous studies have suggested that the expression profiles of miRNAs varied according to various molecular subtypes and exhibit some relations with prognosis. ${ }^{82} \mathrm{BCSCs}$ widely exist in various subtypes of breast cancer cell lines. In fundamental studies, most BCSCs were extracted from multiple subtypes of breast cancer cell lines such as MCF-7, SKBR-3, and MDA-MB-3 according to molecular markers with flow cytometry. Compared to breast cancer cells, miRNAs have broader effects on BCSCs. For instance, let-7c and let-7f are mostly abnormally expressed in luminal A breast cancer, which is different from other molecular 
subtypes, whereas the abnormal expression of let-7 in BCSCs has certain impact upon several cell lines (including MCF-7, MDA-MB231, and SKBR-3), thus affecting the biologic behaviors of breast cancer. ${ }^{83}$ Apart from luminal A, triplenegative and HER2-positive types are also included in these cell lines, perhaps because BCSCs do not come from normal BCSCs, but from the acquisition of stemness by breast cancer cells. Hence, targeted attack of BCSCs by miRNAs would be more effective than the direct attack of breast cancer cells.

Breast cancer drug resistance is one of the important causes that inhibit the prognosis of patients with breast cancer. These years, researches on miRNA and breast cancer drug resistance have been growing, whereas there is still a relative lack of reports on miRNA regulation or even reversion of drug resistance of BCSCs. Drug-resistant breast cancer cells have more or less some stem cell traits, and the number of BCSCs increases significantly after treatment. As a result, breast cancer becomes more malignant and drug resistant. Certain potent anticancer miRNAs related to stem cells, including miR-200 and let-7, with potential effects for increasing chemotherapy sensitivity, are likely to become a tool for predicting drug resistance. At present, lots of molecules and drugs for regulating signal pathways of breast cancer are being tested in clinical practices, whereas miRNAs are still explored in subclinical animal experiments only. This may be due to the complicated mechanisms of BCSCs. Further, which miRNA should be chosen, how to precisely transform miRNAs into BCSCs, and whether the transferred miRNAs or miRNA inhibitors also impact normal cells all remain unanswered. In the future, highly specific smallmolecule drugs are expected to regulate miRNA activity and potentially eradicate BCSCs to treat breast cancer.

\section{Acknowledgment}

This study was supported by a foundation for the 1022 first level of innovative talents of Zhejiang Cancer Hospital, China (grant number 2013102202), and Key platform technological project of Zhejiang medical science and hygiene (grant number 2016ZDB003).

\section{Author contributions}

All authors contributed toward data analysis, drafting and revising the paper and agree to be accountable for all aspects of the work.

\section{Disclosure}

The authors report no conflicts of interest in this work.

\section{References}

1. Williams C, Helguero L, Edvardsson K, et al. Gene expression in murine mammary epithelial stem cell-like cells shows similarities to human breast cancer gene expression. Breast Cancer Res. 2009;11:R26.

2. Shackleton M, Vaillant F, Simpson KJ, et al. Generation of a functional mammary gland from a single stem cell. Nature. 2006;439: $84-88$.

3. Friederichs J, Zeller Y, Hafezi-Moghadam A, et al. The CD24/P-selectin binding pathway initiates lung arrest of human A125 adenocarcinoma cells. Cancer Res. 2000;60:6714-6722.

4. Thomas SN, Zhu F, Schnaar RL, Alves CS, Konstantopoulos K. Carcinoembryonic antigen and CD44 variant isoforms cooperate to mediate colon carcinoma cell adhesion to E- and L-selectin in shear flow. J Biol Chem. 2008;283:15647-15655.

5. Ginestier C, Hur MH, Charafe-Jauffret E, et al. ALDH1 is a marker of normal and malignant human mammary stem cells and a predictor of poor clinical outcome. Cell Stem Cell. 2007;1:555-567.

6. Gupta PB, Fillmore CM, Jiang G, et al. Stochastic state transitions give rise to phenotypic equilibrium in populations of cancer cells. Cell. 2011;146:633-644.

7. Pece S, Tosoni D, Confalonieri S, et al. Biological and molecular heterogeneity of breast cancers correlates with their cancer stem cell content. Cell. 2010;140:62-73.

8. Liu S, Wicha MS. Targeting breast cancer stem cells. J Clin Oncol. 2010;28:4006-4012.

9. Li X, Lewis MT, Huang J, et al. Intrinsic resistance of tumorigenic breast cancer cells to chemotherapy. J Natl Cancer Inst. 2008; 100:672-679.

10. Creighton CJ, Li X, Landis M, et al. Residual breast cancers after conventional therapy display mesenchymal as well as tumor-initiating features. Proc Natl Acad Sci U S A. 2009;106:13820-13825.

11. Ha M, Kim VN. Regulation of microRNA biogenesis. Nat Rev Mol Cell Biol. 2014;15:509-524.

12. Bertoli G, Cava C, Castiglioni I. MicroRNAs: new biomarkers for diagnosis, prognosis, therapy prediction and therapeutic tools for breast cancer. Theranostics. 2015;5:1122-1143.

13. Aydogdu E, Katchy A, Tsouko E, et al. MicroRNA-regulated gene networks during mammary cell differentiation are associated with breast cancer. Carcinogenesis. 2012;33:1502-1511.

14. Takahashi RU, Miyazaki H, Ochiya T. The roles of MicroRNAs in breast cancer. Cancers (Basel). 2015;7:598-616.

15. Lee YC, Chang WW, Chen YY, et al. Hsp $90 \alpha$ mediates BMI1 expression in breast cancer stem/progenitor cells through facilitating nuclear translocation of c-Myc and EZH2. Int J Mol Sci. 2017;18(9):1986.

16. Shimono Y, Zabala M, Cho RW, et al. Downregulation of miRNA200c links breast cancer stem cells with normal stem cells. Cell. 2009;138:592-603.

17. Kawaguchi T, Yan L, Qi Q, et al. Overexpression of suppressive microRNAs, miR-30a and miR-200c are associated with improved survival of breast cancer patients. Sci Rep. 2017;7:15945.

18. Song C, Liu LZ, Pei XQ, et al. miR-200c inhibits breast cancer proliferation by targeting KRAS. Oncotarget. 2015;6:34968-34978.

19. Lim YY, Wright JA, Attema JL, et al. Epigenetic modulation of the miR-200 family is associated with transition to a breast cancer stemcell-like state. J Cell Sci. 2013;126:2256-2266.

20. Qian P, Banerjee A, Wu ZS, et al. Loss of SNAIL regulated miR128-2 on chromosome 3p22.3 targets multiple stem cell factors to promote transformation of mammary epithelial cells. Cancer Res. 2012;72:6036-6050.

21. Li B, Lu Y, Wang H, et al. miR-221/222 enhance the tumorigenicity of human breast cancer stem cells via modulation of PTEN/Akt pathway. Biomed Pharmacother. 2016;79:93-101.

22. Yang Z, Han Y, Cheng K, et al. miR-99a directly targets the mTOR signalling pathway in breast cancer side population cells. Cell Prolif. 2014;47:587-595. 
23. Guo F, Chen H, Chang J, Zhang L. Mutation R273H confers p53 a stimulating effect on the IGF-1R-AKT pathway via miR-30a suppression in breast cancer. Biomed Pharmacother. 2016;78:335-341.

24. Ouzounova M, Vuong T, Ancey PB, et al. MicroRNA miR-30 family regulates non-attachment growth of breast cancer cells. BMC Genomics. 2013;14:139.

25. Czerwinska P, Kaminska B. Regulation of breast cancer stem cell features. Contemp Oncol (Pozn). 2015;19:A7-A15.

26. Harrison H, Farnie G, Brennan KR, Clarke RB. Breast cancer stem cells: something out of notching? Cancer Res. 2010;70:8973-8976.

27. Hoey T, Yen WC, Axelrod F, et al. DLL4 blockade inhibits tumor growth and reduces tumor-initiating cell frequency. Cell Stem Cell. 2009;5: $168-177$.

28. Wolfson B, Eades G, Zhou Q. Roles of microRNA-140 in stem cell-associated early stage breast cancer. World J Stem Cells. 2014;6:591-597.

29. Drago-Ferrante R, Pentimalli F, Carlisi D, et al. Suppressive role exerted by microRNA-29b-1-5p in triple negative breast cancer through SPIN1 regulation. Oncotarget. 2017;8:28939-28958.

30. Yu F, Yao H, Zhu P, et al. let-7 regulates self renewal and tumorigenicity of breast cancer cells. Cell. 2007;131:1109-1123.

31. Piskounova E, Polytarchou C, Thornton JE, et al. Lin28A and Lin28B inhibit let-7 microRNA biogenesis by distinct mechanisms. Cell. 2011; 147:1066-1079.

32. Cai WY, Wei TZ, Luo QC, et al. The Wnt-beta-catenin pathway represses let-7 microRNA expression through transactivation of Lin 28 to augment breast cancer stem cell expansion. J Cell Sci. 2013;126: 2877-2889.

33. Aceto N, Sausgruber N, Brinkhaus H, et al. Tyrosine phosphatase SHP2 promotes breast cancer progression and maintains tumor-initiating cells via activation of key transcription factors and a positive feedback signaling loop. Nat Med. 2012;18:529-537.

34. El HR, Pinna G, Cabaud O, et al. miR-600 acts as a bimodal switch that regulates breast cancer stem cell fate through WNT signaling. Cell Rep. 2017;18:2256-2268.

35. Bodal VK, Sangwan S, Bal MS, et al. Association between Microrna 146a and Microrna 196a2 Genes Polymorphism and Breast Cancer Risk in North Indian Women. Asian Pac J Cancer Prev. 2017;18: 2345-2348.

36. Chernyi VS, Tarasova PV, Kozlov VV, et al. Search of MicroRNAs regulating the receptor status of breast cancer In Silico and experimental confirmation of their expression in tumors. Bull Exp Biol Med. 2017;163: 655-659.

37. Petrovic N, Davidovic R, Bajic V, Obradovic M, Isenovic RE. MicroRNA in breast cancer: the association with BRCA1/2. Cancer Biomark. 2017; 19:119-128.

38. Li Y, Xu Y, Yu C, Zuo W. Associations of miR-146a and miR-146b expression and breast cancer in very young women. Cancer Biomark. 2015;15:881-887.

39. Shimono Y, Mukohyama J, Nakamura S, Minami H. MicroRNA regulation of human breast cancer stem cells. J Clin Med. 2015;5(1):2.

40. Takebe N, Harris PJ, Warren RQ, Ivy SP. Targeting cancer stem cells by inhibiting Wnt, Notch, and Hedgehog pathways. Nat Rev Clin Oncol. 2011;8:97-106.

41. McGowan PM, Simedrea C, Ribot EJ, et al. Notch1 inhibition alters the CD44hi/CD24lo population and reduces the formation of brain metastases from breast cancer. Mol Cancer Res. 2011;9:834-844.

42. Kang L, Mao J, Tao Y, et al. MicroRNA-34a suppresses the breast cancer stem cell-like characteristics by downregulating Notch1 pathway. Cancer Sci. 2015;106:700-708.

43. Yu F, Jiao Y, Zhu Y, et al. MicroRNA 34c gene down-regulation via DNA methylation promotes self-renewal and epithelial-mesenchymal transition in breast tumor-initiating cells. J Biol Chem. 2012;287: 465-473.

44. Mohammadi-Yeganeh S, Mansouri A, Paryan M. Targeting of miR9/ NOTCH1 interaction reduces metastatic behavior in triple-negative breast cancer. Chem Biol Drug Des. 2015;86:1185-1191.
45. Luo J, Yin X, Ma T, Lu J. Stem cells in normal mammary gland and breast cancer. Am J Med Sci. 2010;339:366-370.

46. Kreso A, Dick JE. Evolution of the cancer stem cell model. Cell Stem Cell. 2014;14:275-291.

47. Yu F, Deng H, Yao H, et al. Mir-30 reduction maintains self-renewal and inhibits apoptosis in breast tumor-initiating cells. Oncogene. 2010;29: 4194-4204.

48. Munoz P, Iliou MS, Esteller M. Epigenetic alterations involved in cancer stem cell reprogramming. Mol Oncol. 2012;6:620-636.

49. Pandit TS, Kennette W, Mackenzie L, et al. Lymphatic metastasis of breast cancer cells is associated with differential gene expression profiles that predict cancer stem cell-like properties and the ability to survive, establish and grow in a foreign environment. Int J Oncol. 2009;35:297-308.

50. Reya T, Morrison SJ, Clarke MF, Weissman IL. Stem cells, cancer, and cancer stem cells. Nature. 2001;414:105-111.

51. Thiery JP. Epithelial-mesenchymal transitions in tumour progression. Nat Rev Cancer. 2002;2:442-454.

52. Mani SA, Guo W, Liao MJ, et al. The epithelial-mesenchymal transition generates cells with properties of stem cells. Cell. 2008;133:704-715.

53. Jain $P$, Alahari SK. Breast cancer stem cells: a new challenge for breast cancer treatment. Front Biosci (Landmark Ed). 2011;16:1824-1832.

54. Park SM, Gaur AB, Lengyel E, Peter ME. The miR-200 family determines the epithelial phenotype of cancer cells by targeting the E-cadherin repressors ZEB1 and ZEB2. Genes Dev. 2008;22:894-907.

55. Gregory PA, Bert AG, Paterson EL, et al. The miR-200 family and miR-205 regulate epithelial to mesenchymal transition by targeting ZEB1 and SIP1. Nat Cell Biol. 2008;10:593-601.

56. Eades G, Yao Y, Yang M, Zhang Y, Chumsri S, Zhou Q. miR-200a regulates SIRT1 expression and epithelial to mesenchymal transition (EMT)-like transformation in mammary epithelial cells. J Biol Chem. 2011;286:25992-26002.

57. Tordonato C, Di Fiore PP, Nicassio F. The role of non-coding RNAs in the regulation of stem cells and progenitors in the normal mammary gland and in breast tumors. Front Genet. 2015;6:72.

58. Chang CJ, Chao CH, Xia W, et al. p53 regulates epithelial-mesenchymal transition and stem cell properties through modulating miRNAs. Nat Cell Biol. 2011;13:317-323.

59. Iliopoulos D, Lindahl-Allen M, Polytarchou C, Hirsch HA, Tsichlis PN, Struhl K. Loss of miR-200 inhibition of Suz12 leads to polycombmediated repression required for the formation and maintenance of cancer stem cells. Mol Cell. 2010;39:761-772.

60. Han M, Wang Y, Liu M, et al. MiR-21 regulates epithelial-mesenchymal transition phenotype and hypoxia-inducible factor-1alpha expression in third-sphere forming breast cancer stem cell-like cells. Cancer Sci. 2012;103:1058-1064.

61. Han M, Liu M, Wang Y, et al. Antagonism of miR-21 reverses epithelialmesenchymal transition and cancer stem cell phenotype through AKT/ ERK1/2 inactivation by targeting PTEN. PLoS One. 2012;7:e39520.

62. Han M, Liu M, Wang Y, et al. Re-expression of miR-21 contributes to migration and invasion by inducing epithelial-mesenchymal transition consistent with cancer stem cell characteristics in MCF-7 cells. Mol Cell Biochem. 2012;363:427-436.

63. Song SJ, Poliseno L, Song MS, et al. MicroRNA-antagonism regulates breast cancer stemness and metastasis via TET-family-dependent chromatin remodeling. Cell. 2013;154:311-324.

64. Wang Z, Liao H, Deng Z, et al. miRNA-205 affects infiltration and metastasis of breast cancer. Biochem Biophys Res Commun. 2013;441: 139-143.

65. Chao $\mathrm{CH}$, Chang CC, Wu MJ, et al. MicroRNA-205 signaling regulates mammary stem cell fate and tumorigenesis. J Clin Invest. 2014;124:3093-3106.

66. Hwang-Verslues WW, Chang PH, Wei PC, et al. miR-495 is upregulated by E12/E47 in breast cancer stem cells, and promotes oncogenesis and hypoxia resistance via downregulation of E-cadherin and REDD1. Oncogene. 2011;30:2463-2474. 
67. Liu S, Patel SH, Ginestier C, et al. MicroRNA93 regulates proliferation and differentiation of normal and malignant breast stem cells. PLoS Genet. 2012;8:e1002751.

68. Patel JS, Hu M, Sinha G, et al. Non-coding RNA as mediators in microenvironment-breast cancer cell communication. Cancer Lett. 2016;380:289-295.

69. Kaminska B, Kulesza DW, Ramji K. Overview of mechanisms of cancer stem cell drug resistance. Curr Signal Transduct Ther. 2013;8: $180-192$.

70. Parasramka MA, Ho E, Williams DE, Dashwood RH. MicroRNAs, diet, and cancer: new mechanistic insights on the epigenetic actions of phytochemicals. Mol Carcinog. 2012;51:213-230.

71. Zhu Y, Yu F, Jiao Y, et al. Reduced miR-128 in breast tumor-initiating cells induces chemotherapeutic resistance via Bmi-1 and ABCC5. Clin Cancer Res. 2011;17:7105-7115.

72. Zhang X, Wan G, Mlotshwa S, et al. Oncogenic Wip1 phosphatase is inhibited by miR-16 in the DNA damage signaling pathway. Cancer Res. 2010;70:7176-7186.

73. Melo SA, Sugimoto H, O'Connell JT, et al. Cancer exosomes perform cell-independent microRNA biogenesis and promote tumorigenesis. Cancer Cell. 2014;26:707-721.

74. Lee JK, Park SR, Jung BK, et al. Exosomes derived from mesenchymal stem cells suppress angiogenesis by down-regulating VEGF expression in breast cancer cells. PLoS One. 2013;8:e84256.

75. Ono M, Kosaka N, Tominaga N, et al. Exosomes from bone marrow mesenchymal stem cells contain a microRNA that promotes dormancy in metastatic breast cancer cells. Sci Signal. 2014;7:a63.
76. Lim PK, Bliss SA, Patel SA, et al. Gap junction-mediated import of microRNA from bone marrow stromal cells can elicit cell cycle quiescence in breast cancer cells. Cancer Res. 2011;71:1550-1560.

77. Yang M, Chen J, Su F, et al. Microvesicles secreted by macrophages shuttle invasion-potentiating microRNAs into breast cancer cells. Mol Cancer. 2011;10:117.

78. Baglio SR, Rooijers K, Koppers-Lalic D, et al. Human bone marrowand adipose-mesenchymal stem cells secrete exosomes enriched in distinctive miRNA and tRNA species. Stem Cell Res Ther. 2015; 6:127.

79. Vallabhaneni KC, Penfornis P, Dhule S, et al. Extracellular vesicles from bone marrow mesenchymal stem/stromal cells transport tumor regulatory microRNA, proteins, and metabolites. Oncotarget. 2015; 6:4953-4967.

80. Liu J, Meng T, Yuan M, et al. MicroRNA-200c delivered by solid lipid nanoparticles enhances the effect of paclitaxel on breast cancer stem cell. Int J Nanomedicine. 2016;11:6713-6725.

81. Barh D, Malhotra R, Ravi B, Sindhurani P. MicroRNA let-7: an emerging next-generation cancer therapeutic. Curr Oncol. 2010;17:70-80.

82. van Schooneveld E, Wildiers H, Vergote I, Vermeulen PB, Dirix LY, Van Laere SJ. Dysregulation of microRNAs in breast cancer and their potential role as prognostic and predictive biomarkers in patient management. Breast Cancer Res. 2015;17:21.

83. Peng F, Li TT, Wang KL, et al. H19/let-7/LIN28 reciprocal negative regulatory circuit promotes breast cancer stem cell maintenance. Cell Death Dis. 2017;8:e2569.
OncoTargets and Therapy

\section{Publish your work in this journal}

OncoTargets and Therapy is an international, peer-reviewed, open access journal focusing on the pathological basis of all cancers, potential targets for therapy and treatment protocols employed to improve the management of cancer patients. The journal also focuses on the impact of management programs and new therapeutic agents and protocols on

\section{Dovepress}

patient perspectives such as quality of life, adherence and satisfaction. The manuscript management system is completely online and includes a very quick and fair peer-review system, which is all easy to use. Visit http://www.dovepress.com/testimonials.php to read real quotes from published authors. 